\title{
The Role of Case Studies in Effective Data Sharing, Reuse and Impact
}

\author{
by Rebecca Parsons ${ }^{1}$ and Scott Summers ${ }^{2}$
}

\begin{abstract}
The effectiveness and impact of social science research is under constant review. From the sharing, reuse and archiving of social science research data to the outcomes, reach and impact of research, social science professionals are under increasing pressure to realise the maximum potential of their data collections and their research findings. The UK Data Service is playing a key role in supporting researchers in this process and is using detailed and well-received case studies to provide them with guidance on the best practice for sharing and reusing data and also identifying and capturing the impact of research. The impact of research is now routinely considered when examining the 'success' of funded projects, but the reality of identifying and capturing impact can be a challenge. Publishing data in its own right is now recognised as being impactful to funders, yet exposing this narrative through 'showcasing' is under exploited. Such narratives can incentivise others to share data and also improve the quality of the data and documentation. This paper seeks to explore the role that case studies of research can play in this regard. The paper also examines the role that depositor and user case studies can play in enhancing the reuse of a showcased data collection. To achieve this, a variety of illustrative depositor and impact case studies are discussed, highlighting the role that these can have on research projects.
\end{abstract}

\section{Keywords}

Case Study, Impact, Data Sharing, Data Management.

\section{Introduction}

This paper explores the use of case studies in a variety of ways; First, it considers cases studies in regards to research and impact. Second, the role they can play in assisting other depositors with depositing data at the UK Data Service. Third, the role they can play in brand recognition and impact for the UK Data Service. Finally, the paper concludes by identifying areas where we can develop the use of case studies further in the future.

The UK Data Service has for a number of years been producing case studies on the data that we hold. ${ }^{3}$ These have traditionally been interviews or short vignettes of research papers, linked to the catalogue record of the data, and were mainly intended to show other researchers how particular data had been used. These short case studies described the research question posed, the data used, the methodology applied to the research and the findings and publications produced by the researcher. Appendix 1 gives an example of this type of case study.

Case studies of this type are used primarily to give UK Data Service users accessing data information about research that had already used these data. They provide the basic 'narrative' for a piece of research, but do not look further at whether the research has been applied in practice, whether it has influenced policy or decision making, or whether any follow-up work has been completed.

Over the past few years' we have been looking in more detail at how the UK Data Service is used and have been starting to track the impact of research using data held in the collection. A small team was created in the UK Data Service Communications section, under the guidance of a Director for Communications and Impact, which was tasked to identify and track research impact. Identifying research impact is a time-consuming process and the team at the UK Data Service uses several methods to identify impactful research. The team follow the review documents produced for the national UK government and for local and regional government, policy reviews prepared by several leading UK think tanks and data use in the media. From these we can identify the research 
publications that have been used for evidence and, if it has come from our collection, track the data use back to the UK Data Service. As well as this backwards identification of impact, the team are also in contact with think tanks and individual researchers about work that they are currently undertaking, which may be impactful in the future. This allows us to follow individual projects as they are happening. We also encourage researchers to submit details of publications and the team can then search to see if they have been used in policy.

The focus on research impact is a relatively recent development in the Higher Education sector of the UK and has moved the definition of 'successful' research beyond simple metrics (for example a paper has been cited ' $X$ ' number of times, or a dataset has been downloaded ' $X$ 'times), to attempting to define how research has been used and what practical benefits it has brought. This change in measuring successful research has occurred at the same time as the expansion in providing open data, which anyone can download and use. For data which requires users to register with the UK Data Service, it is possible to track the metrics of data use, but for open data we do not record in detail how data are being used. There is now a greater emphasis on making data as open as practicable to allow greater use and reuse, but the focus of funders, research councils and the government is now not on the quantity of data being accessed, but on the quality of the research that comes from the data. Case studies allow us to record the quality of research.

We are still producing shorter case studies to document research, but we are supplementing these with case studies focussing on the impact that research has in the wider world, as well as the role that the UK Data Service plays in developing tools for data use and that share depositor stories for the benefit of other researchers. An example of an 'Impact'case study can be found in Appendix 2. The process of developing these case studies has been eye-opening, not just in seeing how widely the UK Data Service is used and the breadth of research that is coming from the data deposited with us and downloaded from us, but has also given us a greater understanding of the issues facing researchers in using and sharing data. Case studies have enabled us to spread the word about the work that we do, but also analyse how the Service is used and where we can improve our support for data users.

\section{Research and impact}

A key feature of case studies is that they allow us to demonstrate the reach and impact of the research that is carried out using data downloaded from, or deposited with, the UK Data Service. Within the UK there is a clear need for researchers to demonstrate the impact of their work, with 'impact' being a key indicator of success when universities are graded under the Research Excellence Framework (REF) a national system for assessing the quality of research produced by UK institutions. ${ }^{4}$ The REF uses case studies as a method of comparing the research output from universities and institutions who have to submit detailed case studies as part of their evaluation process. Universities were last graded by the REF in 2014 and are now familiar with the developing case studies to show impact. Case studies have become a familiar way of documenting the effect that research and projects have and most universities now employ staff specifically to promote research impact in preparation for the next REF in 2020.

Through talking with researchers that use the UK Data Service, we found that the expansion of these institutional impact case studies came with some issues for researchers. This is sometimes because the research has not yet produced any visible impact at the completion of the project, or because the researcher has moved on to new work and does not have the resources to identify the impact of their past work. This is particularly an issue for early career researchers, who may be building their portfolio of work and moving between academic institutions. UK universities are selective in which researchers are submitted to the REF for assessment, so researchers whose projects do not produce impact in a specific timescale can miss-out on institutional support for documenting the impact of their research. The UK Data Service identified this as one area where we could support researchers that use the Service, providing useful evidence for researchers to use in their own career development and, at the same time, boost the profile and use of the UK Data Service. Researchers can submit papers or projects that have used the Service to us and the impact team will follow the outputs of the research to see what impact there has been. The case study is then prepared giving a summary of the research, information about the data that was used and the impact that has come from it. The case study is then used by the UK Data Service to promote the use of the data and is shared with the researcher for them to use too. The development of impact specific case studies has shown the breadth of research that has been undertaken using data from the UK Data Service. Our case studies come from the fields of health, ${ }^{5}$ social policy, ${ }^{6}$ education, ${ }^{7}$ technology use, $^{8}$ and business. ${ }^{9}$

Examples from our most recent case studies include, Dr Ivy Shiue who carried out a piece of research that compared health bio-markers in older adults with the temperature of their home and showed that living in a colder home has a detrimental effect on a person's health, a finding which was used in the UK government's Winter Planning Policy. ${ }^{10}$ Dr Shiue has worked with the UK Data Service for several years and contacts the impact team when she publishes a piece of work that she thinks could be used by policy makers. Another project entitled 'Onward Migration,' which investigated the experiences of refugees across the UK, was used as evidence by the Scottish Refugee Council on the effect of forced relocation on refugee integration. ${ }^{11}$ This research was identified through media coverage of the project that prompted the impact team to study the data used by the researchers and contact them about their work. As a final example, we even have evidence of energy use for English wine production, a piece of research which led to advice for English wine producers on reducing their energy costs and carbon footprint, ${ }^{12}$ which was identified following the researcher submitting details of their research paper to the Service.

\section{Assisting other depositors through illustrative case studies}

Another use that the UK Data Service has identified for depositor case studies is that of providing assistance to other depositors. Put simply, depositor case studies allow us to point other depositors to illustrative examples of previous users that have deposited data with 
us and how they managed - and dealt with - any challenges they may have had when depositing data. This has been highly informative not just for potential depositors, but also for the team who engage in research data management training.

For example, one recent depositor case study helps to emphasise the message that we have been promoting for years at the UK Data Service, namely: the importance of planning for the sharing and managing of data early within the research project. ${ }^{13}$ Dr Karon Gush's study, which investigated how couples managed their households during recessions did just that, enabling the data to be effectively deposited and shared with the UK Data Service. ${ }^{14}$ Dr Gush and her team identified the potential challenges when it came to archiving and sharing the data early on in the project and sought to address them throughout the project so as to prevent future issues. For example, consent for sharing was gained from the participants when they were interviewed because the researchers had already identified that they wished to share the data and were aware that they would need 'fully informed consent' from the participants to do this. When it came to anonymising the data a careful balancing act had to be found so as to ensure that the data was as useful as it could be to future users, whilst still achieving the correct level of anonymity for participants. Dr Gush put it best in the case study interview when she said:

"Anonymisation should be thought about at the beginning and should be seen as 'part and parcel' of the whole project... and the anonymisation process should be completed as you go along and not left until the end." ${ }^{15}$

This is an important message that we have always promoted. Now we can easily point depositors and users to the case study to highlight this.

Other depositor case studies have allowed us to explore and discuss a variety of other challenges; such as, the problems with archiving complex anthropological data ten years after the research is completed, ${ }^{16}$ and how one can gain consent for data sharing and archiving retrospectively. ${ }^{17}$

These case studies also help to illustrate the importance of adequate data management training, and planning being undertaken at the beginning of the depositor's research project. By doing so, it ensures that most challenges/issues related to the research, the data, sharing and archiving are identified early on. Thus, potential issues can be adequately addressed, which in turn leads to better quality sharable data. Alongside this, there is a saving of resources for the researcher (both in terms of finance and time).

Utilising depositor case studies in this way has a variety of core benefits for us, our depositors and, our users; Firstly, it allows us to actively encourage data deposits from users by showing how other data has been deposited and shared. Secondly, it provides illustrative examples of how other depositors have addressed potential data sharing issues in practice. Thirdly, it allows data depositors to build collaborations with users by providing further information on their research project and the steps that they went through to deposit their data. For example, this might be the anonymisation or consent procedure(s) they followed. Fourthly (and finally), it allows us as a Service to have impact in a variety of ways. Specifically, it allows us to help other researchers to deposit data with us, share their research and experiences, have additional impact with their research, and provides examples of impact for our funders.

\section{Raising awareness of the UK Data Service}

The final benefit to developing a range of case studies has been the collating of real-world evidence on the work of the UK Data Service. As with any publically funded organisation, the Service has a need to prove to our funders and supporters the usefulness of the work that we do. In many ways, 'data' and 'data archiving' can be rather vague terms, particularly for those who do not work in the archiving or social science areas, and explaining the breadth of the work of the UK Data Service to people in the world outside our niche becomes much easier if we can show real examples of the data we hold actually in use. The UK Data Service is funded by the Economic and Social Research Council (ESRC) and they have a remit to share the work that they fund with the general public. ${ }^{18}$ The case studies that we have developed for our funders focus both on individual pieces of research using data from our collection and also specific studies on how the Service is being used. These more technical case studies look at the expertise and technology that the UK Data Service itself has and provide evidence for how the Service is used. An example of this would be our case study on InFuse the interface that provides access to Census data. ${ }^{19}$ We developed this case study to show how InFuse was collaboratively developed, who was currently using it and some examples of research that had made use of it. This case study is now used by the ESRC to illustrate the benefit of supporting data infrastructure like the UK Data Service. ${ }^{20}$

For use outside the data archiving community we worked with a data visualisation company to develop visual case studies, based on our impact and research case studies. These are an attempt to distil - on to one page - the research question, findings, methodology and policy implications of a piece of research, for example this case study looks at how long it takes elderly people to cross the road:

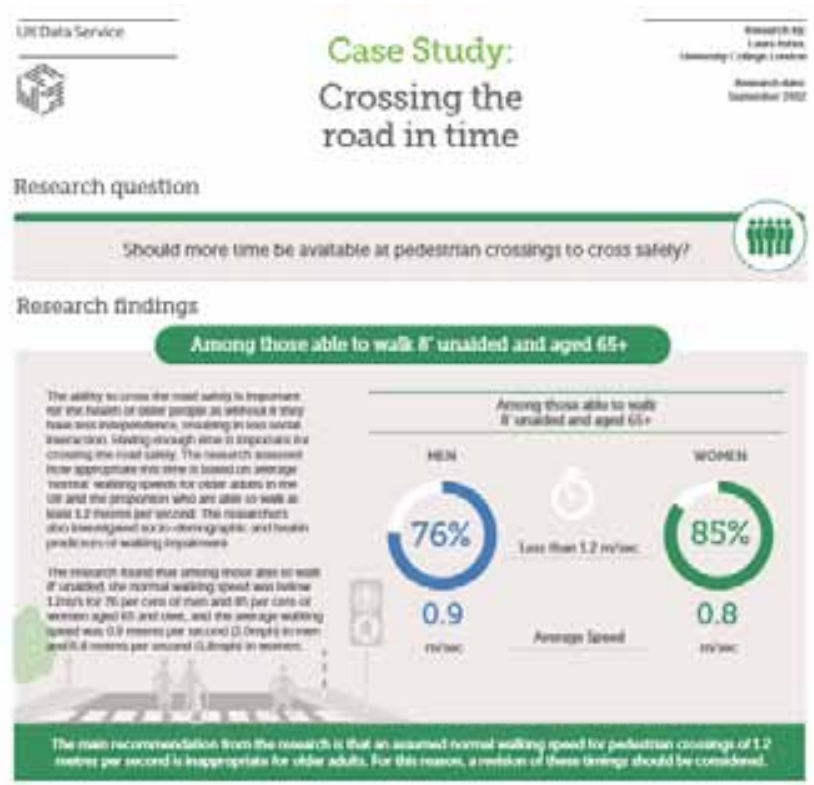


This form of case study does have limitations: it is not suitable for very complex research questions and care needs to be taken that results or methodology are not over simplified by the visual representation. However, we have found these simple case studies useful when introducing the work of the Service, particularly for school students aged 16-18 years old and undergraduate students, who are just starting to use social science data for themselves and need an entry level summary of how some of the data is being used.

\section{Conclusions and the future}

In the future we would like to continue to engage with new UK Data Service users by developing case studies specifically for use in schools that can link to datasets suitable for teaching and are based on UK education curriculum. We also plan to develop case studies specifically focussing on early-career researchers, show-casing their work and helping them build a portfolio of impactful research. We will be continuing to add to our case study collection, with a particular focus on the innovative use of data.

In addition, we plan to further develop the use of depositor case studies to assist our other depositors and complement our current research data management training and guidance. In particular, we will be identifying depositors' collections which have 'acutely sensitive data' or are typically seen as 'data that cannot be shared easily', which we can point other users to. We will utilise these not only to illustrate how data can be effectively shared but also how these potential issues can be simply and effectively addressed in practice.

\section{Appendix}

\section{Example Research Case Study}

\section{Health effects of industrial incinerators in England ${ }^{21}$}

About the research: Research specific to waste incinerators has provided mixed evidence for the effects of proximity to incinerators on health. Older incinerators have been associated with increased incidence and mortality from selected cancers, while more recent reports show little association. Despite this, the effect of incinerator emissions remains a public health issue. This study assesses whether living close to industrial incinerators in England is associated with increased risk of cancer incidence and mortality.

The results show no evidence of elevated risk for those living in areas containing an incinerator compared to those living in matched areas without an incinerator. Moreover, within areas, there is little evidence of an increase in risk for those living in close proximity to an incinerator compared to those living further away.

About the data: This research draws on aggregate data from the 2001 Census for England. The data were key to the study for the identification of case circles around each incinerator, and for obtaining Lower Super Output Area level population counts, by five-year age groups and gender, for 2001 .

Methodology: The researchers considered five regions with industrial incinerators in England, compared with five matched control regions, from 1998 to 2008. Spatial and temporal trends in annual health outcome data within each circular region are analysed. Initially, the researchers used a Poisson log-linear model including age-standardised expected count as an offset and covariates for casecontrol status, matched pair and deprivation, fitted to circle level data to investigate temporal trends. They later modelled data at a finer resolution - at the Lower Super Output Area (LSOA) level. A Poisson log-linear model was then used, as previously, with additional covariates for the effect of distance from the incinerator in case areas included as a multiplicative factor. Population data was used in the calculation of the age-standardised expected count.

\section{2.}

\section{Example Impact Case Study}

\section{Do higher energy prices affect international trade?22}

About the research: Dr Misato Sato and Dr Antoine Dechezleprêtre at the Grantham Research Institute on Climate Change and the Environment at the London School of Economics and Political Science have been studying climate change policy and its effects on trade in a research project funded by the European Union Seventh Framework Programme under grant number no. 308481 (ENTRACTE Research Project), the Global Green Growth Institute, the Grantham Foundation and the Economic and Social Research Council (ESRC) through the Centre for Climate Change Economics and Policy.

Emissions trading policies, such as the EU emissions trading system (EU ETS), are regulations implemented by many countries and cities to reduce industrial greenhouse gas emissions cost-effectively. These regulations are a key tool for achieving emissions reduction targets. However, according to the European Commission on Climate Action they can result in carbon leakage and may affect the competitiveness of businesses. In fact, standard trade models suggest that policies that increase energy price put domestic firms at a disadvantage relative to foreign rivals facing lower energy prices. Producers of energy intensive products respond to higher energy prices by producing fewer energy-intensive goods which may lead to a decline in net exports and the partial relocation of production to a region with low energy prices. 
In this study Dr Sato and Dr Dechezleprêtre explored whether, and to what degree, changes in relative energy prices might influence trade and competitiveness. This is the first study to analyse the effect that energy costs have on global trade using historical data on trade and energy prices. It analysed 62 business and industry sectors in 42 countries over a 15-year period, from 1996 to 2011, using data that covers $60 \%$ of global merchandise trade.

Findings showed that changes in relative energy prices have a statistically significant but very small impact on imports. On average, a $10 \%$ increase in the energy price difference between two country-sectors increases imports by $0.2 \%$. The impact is larger for energy-intensive sectors but even within these, the effect is minor - changes in energy price differences across time explain less than $0.01 \%$ of the variation in trade flows. The authors calculated that a $30 \%$ increase in energy prices across Europe would cause exports to fall by only $0.5 \%$ and would increase imports by $0.07 \%$. They concluded that "contrary to some claims, rises in energy prices do not have much effect on the global competitiveness of businesses. Even a sizeable difference in the price of energy relative to the rest of the world has only a very small impact on a country's imports and exports."

Methodology: The researchers estimated the short-term effects of energy price differences on bilateral trade at the sector level using a gravity model. Trade between countries is not only influenced by energy costs but many other factors such as transport costs, labour cost, exchange rates, tariffs, trade agreements, common language and common currencies. The study therefore brings together a variety of datasets to analyse the impact of relative energy prices on trade. The coverage and detailed disaggregation of the data used goes well beyond previous work, allowing the first global ex-post analysis of the relationship between trade and energy prices. Bilateral trade data were taken from the CEPII's BACI database which contains detailed bilateral import and export statistics from the UN Commodity Trade database. The researchers also used a unique and comprehensive dataset of industrial energy price indices at the country and sector levels covering 48 countries and 12 industry sectors for the period 1996 to 2011. The dataset was constructed in Sato et al., 2015 and uses data from the International Energy Agency World Energy Balances, the International Energy Agency Energy Prices and Taxes and the World Bank, as well as other sources. The procedures used to construct the dataset including the methodology developed to reduce missing data-points, are documented in the working paper, as are the full set of original data sources. This dataset is made publicly available for download here. Additionally, the researchers used data on GDP and population obtained from the International Monetary Fund's World Economic Outlook (2012) and data on wages obtained from the United Nations Industrial Development Organization (2011).

Findings for policy: This study finds unique evidence suggesting that concerns about the risks of carbon leakage may have been overplayed. Carbon pricing policies are intended to induce energy intensive industry to reduce carbon emissions by making it more expensive to pollute. However, if the carbon price is set too high, there is a risk that companies will respond by relocating production to regions with lax policies, rather than clean up their production. Whether this 'carbon leakage' occurs is an important issue in the debates around how to design emissions trading schemes, and whether or not leakage occurs is a question in much need of robust empirical evidence.

Impact of the research: The study's findings about the risks of carbon leakage have been included as research evidence in the 'Evaluation of the EU ETS Directive' report carried out by the European Commission in November 2015. The evaluation subsequently informed policy measures implemented by the Commission regarding the revision of the EU ETS Directive, as set out in the framework of measures of the conclusions of the European council in October 2014.

The study has also informed the following reports:

- The OECD Environment Working Paper No. 87, a review of literature on ex post empirical evaluations of the impacts of carbon prices on indicators of competitiveness.

- The OECD Economics Department Working Papers No. 1282, "Do environmental policies affect global value chains?"

- The New Climate Economy Working Paper 'Implementing Effective Carbon Pricing', which was written as a supporting document for the 2015 report of the Global Commission on the Economy and Climate, Seizing the Global Opportunity: Partnerships for Better Growth and a Better Climate

- Methods for evaluating the Performance of Emissions Trading Schemes, a Discussion Paper by Climate Strategies, prepared as part of the project "Evaluation of Emissions Trading Scheme Pilots in China"funded by Children's Investment Fund Foundation (CIFF) and executed by the Tsinghua University.

\section{Notes}

1. Rebecca Parsons, UK Data Service, UK Data Archive, University of Essex, Colchester, Essex, UK. rparsons@essex.ac.uk

2. Dr Scott Summers, UK Data Service, UK Data Archive, University of Essex, Colchester, Essex, UK. ssummers@essex.ac.uk

3. UK Data Service (2016), 'Case Studies' https://www.ukdataservice.ac.uk/use-data/data-in-use/case-studies

4. Research Excellence Framework (2014), 'REF 2014' http://www.ref.ac.uk/

5. UK Data Service (2016), 'Nearly a third of Welsh adults struggling to cope with the pain of chronic health conditions' https://www.ukdataservice. ac.uk/use-data/data-in-use/case-study/?id=192

6. UK Data Service (2015), 'Children with psychological distress are more likely to become unemployed'https://www.ukdataservice.ac.uk/use-data/ data-in-use/case-study/?id=168

7. UK Data Service (2015), 'Exploring the 'middle' in GCSE attainment'https://www.ukdataservice.ac.uk/use-data/data-in-use/case-study/?id=179 
8. UK Data Service (2015), 'Screen-based media and well-being in adolescence' https://www.ukdataservice.ac.uk/use-data/data-in-use/ case-study/?id=177

9. UK Data Service (2015), 'Investigating external and private benefits from investment in skills and training: UK innovators study' https://www. ukdataservice.ac.uk/use-data/data-in-use/case-study/?id=194

10. UK Data Service (2016), 'The impact of cold homes on health'https://www.ukdataservice.ac.uk/use-data/data-in-use/case-study/?id=195

11. UK Data Service (2016), 'Moving on? Dispersal policy, onward migration and integration of refugees in the UK' https://www.ukdataservice. ac.uk/use-data/data-in-use/case-study/?id=191

12. UK Data Service (2015), 'Energy use within the English wine production industry' https://www.ukdataservice.ac.uk/use-data/data-in-use/ case-study/?id=176

13. UK Data Service (2016), 'Karon Gush - Depositor Stories'https://www.ukdataservice.ac.uk/deposit-data/stories/gush

14 ibid

15. ibid

16. UK Data Service (2016),'Pat Caplan - Depositor Stories'https://www.ukdataservice.ac.uk/deposit-data/stories/caplan

17. UK Data Service (2016), 'Maggie Mort - Depositor Stories' https://www.ukdataservice.ac.uk/deposit-data/stories/mort

18. Economic and Social Research Council (2016),'ESRC Shaping Society'http://www.esrc.ac.uk/

19. UK Data Service (2011), 'InFuse' http://infuse.ukdataservice.ac.uk/

20. Economic and Social Research Council (2016), 'Opening up census data for research' http://www.esrc.ac.uk/news-events-and-publications/ impact-case-studies/opening-up-census-data-for-research/

21. UK Data Service (2014) 'Health effects of industrial incinerators in England'https://www.ukdataservice.ac.uk/use-data/data-in-use/ case-study/?id=163

22. UK Data Service (2016), 'Do higher energy prices affect international trade?' https://www.ukdataservice.ac.uk/use-data/data-in-use/ case-study/?id=206 